\title{
BMJ Open Antidepressant use during pregnancy and the risk of gestational diabetes mellitus: a nested case-control study
}

\author{
Maëlle Dandjinou, ${ }^{1,2}$ Odile Sheehy, ${ }^{1}$ Anick Bérard ${ }^{1,2}$
}

To cite: Dandjinou M, Sheehy 0 , Bérard A. Antidepressant use during pregnancy and the risk of gestational diabetes mellitus: a nested casecontrol study. BMJ Open 2019;9:e025908. doi:10.1136/ bmjopen-2018-025908

- Prepublication history and additional material for this paper are available online. To view these files, please visit the journal online (http://dx.doi. org/10.1136bmjopen-2018025908).

Received 07 August 2018 Revised 20 June 2019 Accepted 30 July 2019

\section{Check for updates}

(C) Author(s) (or their employer(s)) 2019. Re-use permitted under CC BY-NC. No commercial re-use. See rights and permissions. Published by BMJ.

${ }^{1}$ Research Center, CHU SainteJustine, Montreal, Quebec,

Canada

${ }^{2}$ Faculty of Pharmacy, University of Montreal, Montreal, Quebec, Canada

Correspondence to

Dr Anick Bérard;

anick.berard@umontreal.ca

\section{ABSTRACT}

Objectives The aim of this study was to determine the association between antidepressant (AD) classes, types and duration of use during pregnancy and the risk of gestational diabetes mellitus (GDM).

Design and setting A nested case-control study was conducted within the Quebec Pregnancy Cohort (QPC), a Canadian provincial database which includes data on all pregnancies and children in Quebec from January 1998 to December 2015.

Primary outcome measures Gestational diabetes mellitus.

Participants Cases of GDM were identified after week 20 of pregnancy and randomly matched 1:10 to controls on gestational age at index date (ie, calendar date of GDM) and year of pregnancy. AD exposure was assessed by filled prescriptions between the beginning of pregnancy (first day of last menstrual period) and index date. Conditional logistic regression models were used to estimate crude and adjusted odds ratios (aOR).

Results Among 20905 cases and 209050 matched controls, 9741 (4.2\%) women were exposed to ADs. When adjusting for potential confounders, $A D$ use was associated with an increased risk of GDM (aOR $1.19,95 \% \mathrm{Cl} 1.08$ to 1.30); venlafaxine (aOR $1.27,95 \% \mathrm{Cl} 1.09$ to 1.49 ) and amitriptyline (aOR $1.52,95 \% \mathrm{Cl} 1.25$ to 1.84 ) were also associated with an increased risk of GDM. Moreover, the risk of GDM was increased with longer duration of $A D$ use, specifically for serotonin norepinephrine reuptake inhibitors, tricyclic ADs and combined use of two AD classes. No statistically significant association was observed for selective serotonin reuptake inhibitors. Conclusion The findings suggest that ADs - and specifically venlafaxine and amitriptyline-were associated with an increased risk of GDM.

\section{INTRODUCTION}

Gestational diabetes mellitus (GDM), defined as a carbohydrate intolerance occurring during pregnancy, diagnosed between 24 weeks and 28 weeks of pregnancy, is a major maternal health condition. ${ }^{12}$ Studies report that $1-20 \%$ of pregnant women are affected by GDM worldwide, depending on the population studied and the diagnosis criteria adopted (International Association of Diabetes and Pregnancy Study Groups (IADPSG) criteria or WHO), and around

\section{Strengths and limitations of this study}

This is the first study investigating antidepressant classes and individual drug use during pregnancy and the risk of gestational diabetes owing to the large number of pregnancies included.

- The Quebec pregnancy cohort is one of the largest medico-administrative databases with 17 years of follow-up, which provides prospectively collected data for pregnant women with high validity and no recall bias possible.

- Our analysis, restricted to a subgroup of pregnancies with depression/anxiety before pregnancy, minimises the risk for confounding by indication.

- Even though our database included a large set of variables, some lifestyle variables such as smoking and body mass index are missing therefore residual confounding cannot be completely excluded.

7-9\% of pregnant women are affected in Quebec. ${ }^{3-6}$ Moreover, the number is rising concomitantly with the surge in overweight and obesity. ${ }^{7}$ Pregnancies with GDM are at higher risk of birth complications (eg, macrosomia, caesarian and dystocia) and may predispose offspring to type 2 diabetes and obesity. ${ }^{8-10}$ Furthermore, mothers with a history of GDM are more likely to develop type 2 diabetes and cardiovascular diseases later in life. ${ }^{11}$

Antidepressants (ADs) are commonly used during pregnancy and neurotransmitters such as serotonin, norepinephrine and dopamine are the mainstay of their action. ${ }^{12}$ ADs have several side effects and some of them increase weight. ${ }^{13}$ There is mounting evidence suggesting that $\mathrm{AD}$ classes might be involved in different ways in biological mechanisms such as weight gain, insulin resistance and glucose metabolism dysregulation to induce diabetes. ${ }^{14-17}$ Moreover, both depression and overweight/obesity are prevalent among women of reproductive age. ${ }^{718}$ Discrepant findings have been reported regarding the relationship between depression and body mass index (BMI). 
Some previous studies reported no association, but a meta-analysis reported a possible bidirectional relationship. ${ }^{1920}$ Depression may increase weight gain and obesity through changes in eating patterns or reduced physical activity and biological mechanisms involving neuroendocrine disturbances could explain the association. ${ }^{19}$ Nevertheless, a recent study showed that antenatal depression was not associated with inadequate and excessive gestational weight gain during pregnancy. ${ }^{21}$ As maternal depression could possibly increase weight gain and GDM, the potential confounding effect of underlying maternal depression should be addressed in studies assessing the relationship between $\mathrm{AD}$ use during pregnancy and GDM. Confounding can be controlled at the study design stage (restriction, matching or randomisation) or at the statistical analysis stage (multivariable models). ${ }^{22}$

Two studies in pregnant women have investigated the association between AD use and GDM, but the evidence was inconclusive and some methodological limitations were not considered such as the non-adjustment for maternal depression. ${ }^{23}{ }^{24}$ The first study found that AD use was associated with an increase in the risk of GDM whereas the second showed no association with selective serotonin reuptake inhibitors (SSRIs). The two studies lack information about timing of the GDM diagnosis, and confounding by indication related to maternal depression is possible. The small sample size in both studies did not allow individual drug effects to be considered. Given the increasing use of ADs during pregnancy, the biological evidence and the scarcity of information on the associated risk of GDM, this question urgently needs to be addressed. To our knowledge, no study to date has been designed to explore directly the association between $\mathrm{AD}$ use and the incidence of GDM in pregnancy.

We therefore assessed the impact of overall AD medication, classes and types, as well as duration of $\mathrm{AD}$ exposure on the risk of GDM in a population-based study of pregnant women.

\section{METHODS}

\section{Patient and public involvement}

Patients or the public were not involved in the design of the study.

\section{Source population}

A nested case-control study was conducted within the Quebec Pregnancy Cohort (QPC). The QPC is an ongoing population-based cohort with prospective data collection on all pregnancies insured by the Quebec Public Prescription Drug Insurance Plan, from 1998 to 2015. Individual-level information was obtained from province-wide databases and linked using healthcare unique personal identifiers.

The QPC was established by identifying all pregnancies in the Régie de l'assurance maladie du Québec (RAMQ) and the Quebec hospitalisation archives (MedEcho) databases. The first day of the last menstrual period (first day of gestation, 1DG) was defined using data on gestational age, which was validated against ultrasound measurements in patient charts. Prospective follow-up was available from 1year prior to 1DG, during pregnancy and until December 2015.

Data sources for this study comprised the medical service database (RAMQ: diagnoses and medical procedures), the Quebec Prescription Drug Insurance Database (drug name, start date, dosage and duration), the Hospitalisation Archive Database (MedEcho: in-hospital diagnoses and procedures) and the Quebec Statistics Database (Institut de la statistique du Québec (ISQ): patient sociodemographic information). Descriptive information regarding the QPC is shown in the paper by Bérard and Sheehy. ${ }^{25}$ Validity studies were performed and confirm the accuracy and high quality of the data sources. ${ }^{2627}$

The study was approved by the Sainte-Justine's Hospital Ethics Committee. The Quebec Commission d'accès à l'information authorised database linkages.

\section{Study cohort definition and study design}

All women in the QPC continuously covered by the public prescription drug plan for at least 6 months before and during pregnancy were eligible. Given that GDM occurs after week 20 of gestation, we excluded abortions and miscarriages. We only included singleton pregnancies as multiple pregnancies are a known risk factor of GDM. Women with type 1 and type 2 diabetes were identified between 6 months before pregnancy and up to week 20 of gestation, and were excluded because they are not at risk of GDM and because overt diabetes is often diagnosed early in pregnancy. Similarly, women with a history of GDM have different metabolic profiles and a different baseline risk of GDM compared with women with no history of GDM, and were thus also excluded from the main analysis. This enabled us to quantify the incident risk of GDM for women without a history of GDM. Studies have reported that women with a history of GDM have a faster deterioration in insulin sensitivity and a lower beta cell compensation which continues to deteriorate after pregnancy. ${ }^{28}$

In order to select a homogeneous population at risk of GDM, we also excluded pregnancies in women with cystic fibrosis as well as those who were overweight (body mass index $($ BMI $)>25 \mathrm{~kg} / \mathrm{m}^{2}$ ) or obese (BMI $>30 \mathrm{~kg} /$ $\left.\mathrm{m}^{2}\right) \cdot{ }^{29}$ Women with cystic fibrosis and overweight or obese women are more likely to develop GDM. Hence, women with obesity and cystic fibrosis have been consistently excluded in previous studies with GDM as outcome. ${ }^{30}$ Women with cystic fibrosis are more likely to have pre-pregnancy diabetes ${ }^{31}$ and pre-pregnancy obesity, which are risk factors for GDM. We excluded those women with either obesity or cystic fibrosis in order to have a homogenous population with regard to these risk factors and to allow comparisons with previous studies.

All pregnancies meeting our inclusion criteria during the study period were considered. Moreover, due to the 
time-varying nature of $\mathrm{AD}$ use during pregnancy, using a nested case-control analysis was appropriate. ${ }^{32}$ As well as a time-dependent survival analysis, this method takes into account time-varying exposure but has the ultimate advantage of being computationally more efficient and providing ORs that are unbiased estimators of rate ratios. $^{33} 34$

\section{Definition of GDM cases}

Cases of GDM were defined as pregnant women with a diagnosis of GDM identified using diagnosis codes of the 9th or 10th editions of the International Classification of Diseases (ICD-9: 250.0-250.9, 648.0, 648.8, 790.2, 775.1 or ICD-10: E10-E14, O24, R73.0) or at least one filled prescription for an antidiabetic drug allowed during pregnancy (insulin, glyburide or metformin), both after week 20 of gestation, whichever occurred first (online supplementary table S1). ${ }^{1}$ This definition was used to identify the earliest calendar date of GDM occurrence (EDI). This definition of the outcome was previously used ${ }^{30} 35$ and GDM ICD codes have been associated with high positive and negative predictive values (PPV: $85 \%$ and NPV: $99 \%) .{ }^{36}$ According to the current guidelines in Canada, ${ }^{1}$ GDM is diagnosed between weeks 24 and 28 of gestation. In our study we included a lag time of 3 weeks to capture late diagnoses of GDM. ${ }^{37}$ If the EDI was between week 20 and week 31 of gestation, the calendar date of the diagnosis of GDM or of a prescription of antidiabetic drug was used as the index date. In other cases, when EDI was recorded after week 31 of gestation, we developed an algorithm which determined the index date by adding 217 days (equivalent to 31 weeks of gestation) to the first day of gestation. This algorithm was used to ensure that all cases were included in the study. Indeed, we hypothesised that EDI could be recorded even after 31 weeks of gestation for several reasons. First, it is possible that a pregnant woman was diagnosed with mild GDM before 31 weeks of gestation and received a prescription of an antidiabetic drug later as her diabetes was controlled with non-pharmacological measures. Second, a woman could also be diagnosed with GDM in her medical visit that was not recorded before 31 weeks of gestation because it was not the most serious medical condition at that moment.

\section{Control selection}

Using the nested case-control design and with the low prevalence of exposure to $\mathrm{AD}$ among controls $(4.1 \%)$, we randomly selected 10 controls for each case among those in the risk set (ie, pregnancy that did not have a diagnosis of GDM at the index date) and matched them by gestational age at index date (ie, calendar date of GDM) and year of pregnancy. As a woman could contribute with repeated pregnancies during the study period, cases and controls were matched on the year of pregnancy.

\section{Antidepressant exposure}

Gestational AD use was assessed using the RAMQ Prescription Drug file. Data on AD prescription fillings have been validated against maternal reports within the study population and have a high predictive value (PPV $100 \%$ and NPV $96 \%) .{ }^{27}$

\section{Overall antidepressant exposure}

Overall $\mathrm{AD}$ exposure was identified as having at least one filled prescription from 1DG to index date. Prescriptions filled before the pregnancy and with a duration which included the 1DG were also considered. The overall AD exposure category was compared with the reference category: no $\mathrm{AD}$ exposure during the same period (see online supplementary table S2 for codes of all ADs evaluated).

\section{Antidepressant exposure by drug class}

We considered $\mathrm{AD}$ exposure by class and formed six mutually exclusive comparison groups: (1) selective serotonin reuptake inhibitors (SSRIs), (2) serotonin norepinephrine reuptake inhibitors (SNRIs), (3) tricyclic ADs (TCAs), (4) others (this category includes all the other ADs used). Pregnancies exposed to at least two classes of $\mathrm{AD}$ were classified as (5) combined. No $\mathrm{AD}$ exposure during the relevant period of time was classified as (6) the reference category.

\section{Antidepressant exposure by drug types}

The following nine active categories were studied: (1) citalopram, (2) fluoxetine, (3) fluvoxamine, (4) paroxetine, (5) sertraline, (6) venlafaxine, (7) amitriptyline and (8) others (this category includes all the other ADs used). Pregnancies exposed to more than one AD were classified as (9) combined. No AD exposure (10) between the 1DG and index date was the reference category. The 10 categories were mutually exclusive.

\section{Antidepressant duration of exposure}

Finally, we looked at the duration of exposure to $\mathrm{AD}$ during pregnancy by adding the length of the filled prescriptions in order to calculate the exact number of days covered by the prescriptions. For pregnancies with a combined use of two or more ADs, if the two ADs had the same overlap, only one exposure time was considered. However, if the duration for the two ADs was not completely overlapping, the additional days of both ADs were added to the overlapping time. Consequently, four mutually exclusive categories were defined: (1) short duration ( $\leq 90$ days of AD exposure), (2) medium duration (90 days $<\mathrm{AD}$ exposure $<180$ days), (3) long duration ( $\geq 180$ days of $\mathrm{AD}$ exposure). The reference category was (4) no exposure to $\mathrm{AD}$ in the relevant time period. We also looked at duration of exposure within each $\mathrm{AD}$ class.

\section{Covariates}

Potential confounders considered for all analyses were (1) sociodemographic variables and maternal characteristics on the 1DG including maternal age, receipt of social assistance (yes/no) and area of residence (urban/rural); (2) maternal chronic comorbidities in the 6 months prior to the pregnancy (physician-based diagnoses or filled prescriptions of related medications for chronic 
comorbidities (depression/anxiety, chronic hypertension, asthma and thyroid disorders); physician-based diagnoses of cardiovascular diseases and polycystic ovarian syndrome); (3) healthcare services utilisation and co-medication (physician visits, hospitalisations or emergency department visits, number of other medications used other than ADs and medications related to chronic comorbidities (antipsychotics, benzodiazepines and corticosteroids are included), visits to a psychiatrist within the 6 months prior to pregnancy). All the previous conditions were identified from either diagnoses or disease-specific medications available in our databases (see online supplementary table S3).

\section{Statistical analysis}

Descriptive analyses (t-tests and $\chi^{2}$ tests for continuous and categorical variables, respectively) were performed to describe the study population. Crude and adjusted ORs and $95 \%$ CIs using conditional logistic regression models were calculated. We considered all analyses significant at a $\mathrm{p}$ value $<0.05$ (two-tailed). We conducted all analyses using SAS version 9.3 software (SAS Institute Inc).

Additionally, the following sensitivity analyses were performed:

1. We restricted the study population to women with depression/anxiety prior to pregnancy as identified by either a diagnosis code for depression or anxiety or an $\mathrm{AD}$ prescription within 6 months before the 1DG. For women with both a diagnosis code and $\mathrm{AD}$ use in the 6 months before the 1DG, only one occurrence was taken into account. This method allowed us to take into account potential confounding by indication.

2. According to the guidelines, GDM is diagnosed between 24 and 28 weeks of gestation; therefore, we looked at diagnoses of GDM strictly between week 24 and week 28. ${ }^{1}$ This will minimise a potential non-differential bias that would underestimate the true estimate.

3. We looked at the exposure within the window from the 1DG to 15 days before the index date in order to ensure that the exposure preceded the outcome.

4. As women with a history of GDM may be at risk of GDM in a future pregnancy, we included women with a history of GDM in the cohort and adjusted for this variable to take into account the confounding related to a history of GDM.

5. As a woman could contribute to the study with repeated pregnancies, and these pregnancies within the same woman may be correlated, we performed an additional analysis using Generalised estimate equation (GEE), which is a method used to address intra-subject correlation. ${ }^{38}$ Results obtained from GEE were compared with findings from the main analysis.

6 . In the main analysis, women with obesity and overweight were excluded as obesity/overweight can be a potential confounder and/or effect modifier. We performed the same analysis in the subgroup of women with obesity and overweight.
RESULTS

In a total of 237172 pregnancies from the QPC meeting the study inclusion criteria, $20905(8.8 \%)$ cases of GDM were identified. This prevalence is concordant with the prevalence reported in Quebec. ${ }^{3}$ Among the cases, 1152 $(5.5 \%)$ women were exposed to ADs (figure 1). The characteristics of the cases and their matched controls $(\mathrm{n}=209050)$ are shown in table 1 . Women with GDM were more likely to be older urban residents and to benefit from social assistance. Maternal comorbidities and risk factors for GDM were more prevalent in cases than in controls.

\section{Primary analyses}

When adjusting for potential confounding factors, using ADs during pregnancy was associated with a modest increased risk of GDM compared with non-users during the study period (adjusted OR (aOR) $1.19 ; 95 \%$ CI 1.08 to $1.30,1152$ exposed cases). AD use before $1 \mathrm{DG}$ as well as a history of depression and anxiety were not associated with GDM risk (table 2).

For AD classes specifically, SNRI $(1.27,95 \%$ CI 1.08 to $1.48,230$ exposed cases), TCA (1.47, 95\% CI 1.22 to 1.77 , 143 exposed cases) and combined use of $\geq 2 \mathrm{AD}$ classes (1.38, 95\% CI 1.15 to $1.67,169$ exposed cases) were associated with an increase in the risk of GDM whereas SSRI were not (1.07, 95\% CI 0.96 to $1.20,535$ exposed cases) (online supplementary table S4).

We also observed a duration-effect gradient associated with gestational AD use. Pregnancies with short duration (1.15, 95\% CI 1.03 to $1.28,541$ exposed cases), medium duration (1.17, $95 \%$ CI 1.00 to $1.39,187$ exposed cases) and long duration of $\mathrm{AD}$ exposure (1.29, 95\% CI 1.13 to $1.48,424$ exposed cases) were at increased risk of GDM compared with unexposed pregnancies (online supplementary table S5). This duration-effect relationship was also observed for TCAs, SNRIs and combined use of two $\mathrm{AD}$ classes, although it did not reach statistical significance for some strata due to the small number of exposed cases (table 3).

Regarding individual ADs, venlafaxine (1.27, 95\% CI 1.09 to $1.49,230$ exposed cases), amitriptyline (1.52, 95\% CI 1.25 to $1.84,133$ exposed cases) and combined use of $\geq 2$ ADs (1.31, 95\% CI 1.10 to $1.56,187$ exposed cases) were associated with an increased risk of GDM. For citalopram $(1.11,95 \%$ CI 0.95 to $1.29,242$ exposed cases), paroxetine (1.13, 95\% CI 0.94 to $1.36,154$ exposed cases) and sertraline $(1.01,95 \%$ CI 0.80 to $1.28,83$ exposed cases), no statistically significant association was observed (see online supplementary table S6; figure 2).

\section{Sensitivity analysis}

In the subgroup of 21395 pre-pregnancy depressed women, exposure to ADs during pregnancy also increased the risk of GDM, which is consistent with the main analysis $(1.15,95 \%$ CI 1.00 to $1.33,1002$ exposed cases). When using a different time window for both the exposure and the outcome, our results were also consistent 


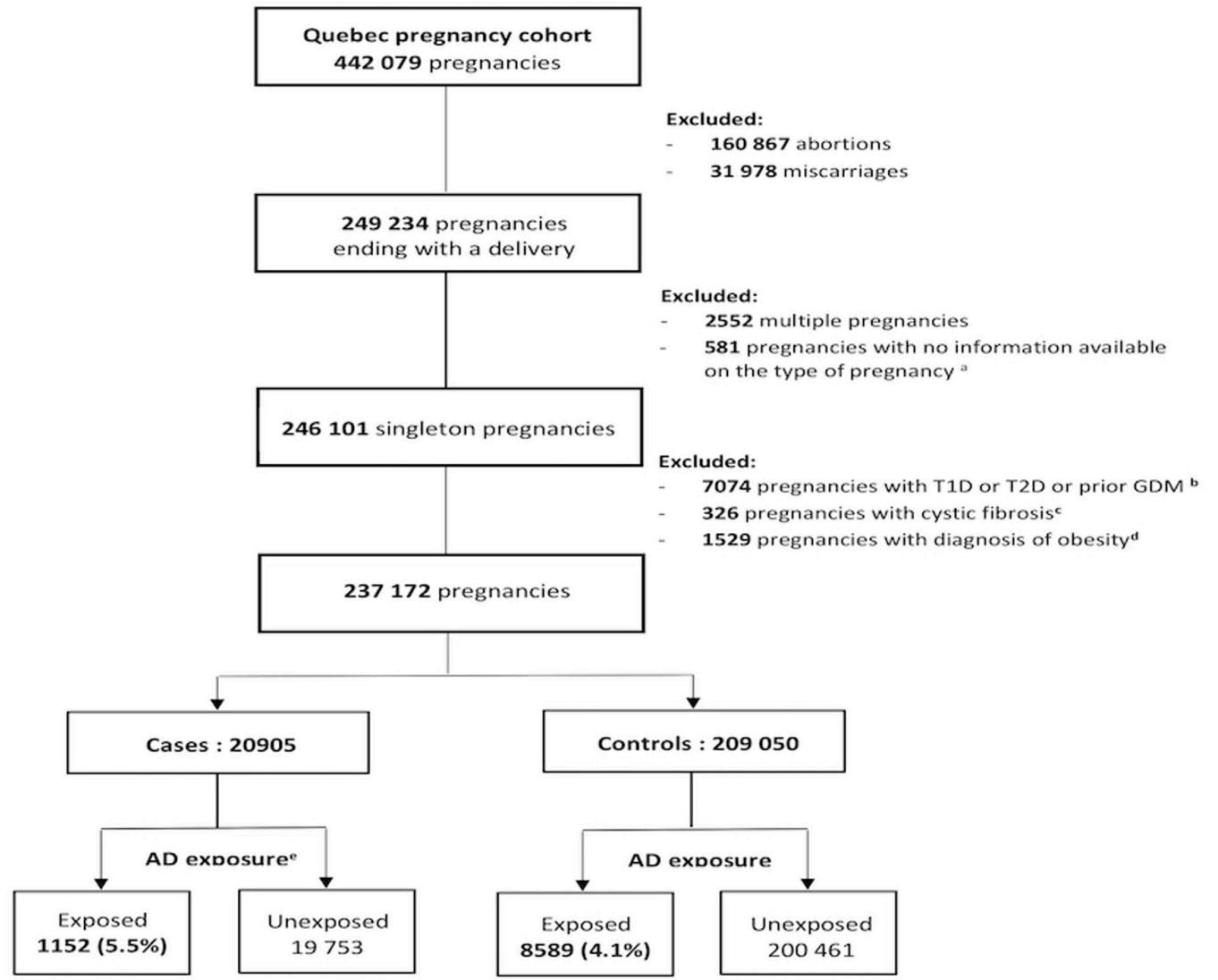

Note: T1D Type 1 Diabetes; T2D Type 2 Diabetes; GDM gestational diabetes mellitus; AD Antidepressant.

a Pregnancies, where we could not assess if gestation was multiple or simple, due to lack of information in databases, were excluded.

b Pregnancies with T1D or T2D or prior GDM identified six months before pregnancy up to week 20 of gestation with ICD $9 / 10$ diagnoses codes: [ICD9: 250.0-250.9, 648.0, 648.8, 790.2, 775.1 or ICD10:E10-E14, O24, R73.0] or a filled prescription for an antidiabetic drug [Insulin or oral hypoglycemic agents : acarbose, gliclazide, glyburide, metformin, pioglitazone, rosiglitazone, sitagliptine, Tolbutamidel.

' Pregnancies with cystic fibrosis identified six months before pregnancy up to week 20 of gestation with ICD $9 / 10$ diagnoses codes: [ICD9: 277.0 or ICD10:E84].

d Pregnancies with a diagnosis of obesity (overweight and obesity combined) identified six months before pregnancy up to week 20 of gestation with ICD 9/10 diagnoses codes: [ICD9: 278.0 or ICD10:E66].

e AD exposure during pregnancy defined by filled prescriptions for Antidepressants between first day of gestation and index date.

Figure 1 Selection of study population: gestational diabetes mellitus.

with the main findings as follows: diagnosis between 24 and 28 weeks only $(1.22,95 \%$ CI 1.01 to $1.48,269$ exposed cases) and exposure between 1DG and 15days before the index date $(1.18,95 \%$ CI 1.08 to $1.30,1139$ exposed cases). When including women with a history of GDM and adjusting for this variable in the model, we found similar results to our main analysis $(1.11,95 \% \mathrm{CI}$ 1.01 to $1.20,1202$ exposed cases). When using the GEE method, which takes into account intra-woman correlation for women contributing to the study with more than one pregnancy, we found similar results to the logistic regression $(1.19,95 \%$ CI 1.09 to 1.31$)$. In the subgroup of women with obesity/overweight, we found higher estimates but the result was not statistically significant due to the sample size $(1.58,95 \%$ CI 0.84 to $2.98,32$ exposed cases) (see online supplementary table $\mathrm{S} 7-12$ ).

\section{DISCUSSION}

In this study, overall $\mathrm{AD}$ use was associated with an increased risk for GDM. Specifically, venlafaxine and amitriptyline were associated with a $27 \%$ and $52 \%$ increased risk of GDM, respectively, after adjustment for major confounders and underlying conditions. No statistically significant association was observed with any SSRI. Moreover, we found a cumulative duration effect for short, medium and long duration of $\mathrm{AD}$ use, which was associated with a $15 \%, 17 \%$ and $29 \%$ increased risk of having GDM, respectively. This effect varied within classes of ADs.

\section{Comparison with other studies}

Literature on this topic is scarce. A previous study by Reis and Källén found that $\mathrm{AD}$ is associated with GDM (OR $1.37,95 \%$ CI 1.08 to 1.75 ), which is consistent with our findings. ${ }^{23}$ For class exposure, our results are also in line with those of Wen $e t a l^{4}$ who reported no association between SSRIs and GDM. In the general non-pregnant population, studies and meta-analyses performed to investigate the risk of type 2 diabetes following $\mathrm{AD}$ use consistently report associations. ${ }^{39-41}$ Conversely, it is still debated whether SSRIs and SNRIs are involved in type 2 diabetes onset, and very few studies have investigated individual drug effects. A meta-analysis by Yoon $e t a t^{41}$ reported the following estimates for individual drugs: paroxetine (risk ratio (RR) 1.52, 
Table 1 Study population characteristics

\begin{tabular}{|c|c|c|c|}
\hline Characteristics & $\begin{array}{l}\text { Cases of GDM } \\
\mathrm{n}=20905\end{array}$ & $\begin{array}{l}\text { Controls } \\
n=209050\end{array}$ & $P$ value \\
\hline \multicolumn{4}{|l|}{ Pregnancy-related variable } \\
\hline Gestational age at index date ${ }^{\star}$, mean (SD) & $29.4(2.3)$ & $29.4(2.3)$ & 1.00 \\
\hline$A D$ exposure from $1 D G$ to index date & $1152(5.5)$ & $8589(4.1)$ & $<0.0001$ \\
\hline$A D$ use during 6 months before gestation & $1448(6.9)$ & $11403(5.4)$ & $<0.0001$ \\
\hline \multicolumn{4}{|c|}{ Sociodemographic characteristics measured on 1DG } \\
\hline \multicolumn{4}{|l|}{ Age (years) } \\
\hline$<18$ & $126(0.6)$ & $2916(1.4)$ & - \\
\hline $18-34$ & $15123(72.3)$ & $173958(83.2)$ & $<0.0001$ \\
\hline$>34$ & $5656(27.1)$ & $32176(15.4)$ & - \\
\hline Urban dwellers & $17678(84.6)$ & $172597(82.6)$ & $<0.0001$ \\
\hline Social assistance recipients & $5704(27.3)$ & $48615(23.3)$ & $<0.0001$ \\
\hline \multicolumn{4}{|c|}{ Maternal comorbidities during 6 months before 1DG } \\
\hline Depression/anxiety§ & $1419(6.8)$ & $12995(6.2)$ & 0.001 \\
\hline Hypertension & $487(2.3)$ & $2665(1.3)$ & $<0.0001$ \\
\hline Asthma† & $1714(8.2)$ & $14637(7.0)$ & $<0.0001$ \\
\hline Cardiovascular diseases§ & $402(1.9)$ & $3468(1.7)$ & 0.004 \\
\hline Thyroid disorders $†$ & $930(4.5)$ & $6354(3.0)$ & $<0.0001$ \\
\hline Polycystic ovarian syndrome§ & $18(0.2)$ & $76(0.1)$ & 0.0007 \\
\hline \multicolumn{4}{|c|}{ Healthcare services utilisation and medication use in the 6 months before 1DG } \\
\hline \multicolumn{4}{|c|}{ No of physician visits } \\
\hline $0-1$ & $10770(51.5)$ & $112674(53.9)$ & $<0.0001$ \\
\hline$\geq 2$ & $10135(48.5)$ & $96376(46.1)$ & - \\
\hline Hospital admission/emergency department visit & $4278(20.5)$ & $42487(20.3)$ & 0.63 \\
\hline \multicolumn{4}{|l|}{ Medications other than ADs $\ddagger$} \\
\hline 0 & $9250(44.2)$ & $95412(45.6)$ & $<0.0001$ \\
\hline $1-2$ & $4800(23.0)$ & $46869(22.4)$ & - \\
\hline$\geq 3$ & $6855(32.8)$ & $66769(32.0)$ & - \\
\hline At least one visit to psychiatrists & $652(3.1)$ & $5523(2.6)$ & $<0.0001$ \\
\hline \multicolumn{4}{|c|}{ Healthcare service utilisation from 1DG until index date } \\
\hline $\begin{array}{l}\text { Pregnancy follow-up by obstetricians/ } \\
\text { gynaecologists }\end{array}$ & $9756(46.7)$ & $77962(37.3)$ & $<0.0001$ \\
\hline
\end{tabular}

*Index date=date of gestational diabetes identification for cases and corresponding date for matched controls.

†Based on ICD-9 or ICD-10 codes or prescriptions filled for hypertension, asthma and thyroid disorders.

łIncluded prescriptions filled for conditions other than depression/anxiety, hypertension, asthma and thyroids disorders.

$\S$ Based on ICD-9 or ICD-10 codes for depression/anxiety, cardiovascular diseases and polycystic ovarian syndrome.

$\mathrm{AD}$, antidepressant; 1DG, first day of gestation; GDM, gestational diabetes mellitus.

95\% CI 0.95 to $2.45,2$ studies) and citalopram (RR 1.13 , $95 \%$ CI 0.85 to 1.49 , 2 studies), which is in line with our results. The relationship between the duration of exposure to ADs and diabetes has been explored in two studies, one in the adult general population and the other in youths. These studies found that the risk of diabetes was increased with a longer duration of use of $\mathrm{AD}$, while taking into account increasing age and weight. ${ }^{42}{ }^{43}$ Similarly, we found that women are at higher risk of GDM with increasing age, and that weight gain during pregnancy could possibly and partly explain the risk of GDM.
Possible biological mechanisms

With respect to biological plausibility, certain mechanisms support the link between AD use and GDM onset. ADs can be involved in diabetes by acting directly on glucose homeostasis, ${ }^{14}$ by decreasing pancreatic insulin secretion, ${ }^{44}$ by increasing cellular insulin resistance ${ }^{45}$ or by acting indirectly on insulin via weight gain. ${ }^{13} 15$

In animal studies it has been reported that ADs can induce hyperglycaemia. ${ }^{45}{ }^{46}$ Moreover, serotonin is involved in glucose homeostasis and is also the target of most ADs, which may explain why ADs affect the glucose 
Table 2 Risk of gestational diabetes following overall AD use during pregnancy

\begin{tabular}{lll}
\hline Variables & $\begin{array}{l}\text { Crude OR } \\
\mathbf{( 9 5 \% ~ C l )}\end{array}$ & $\begin{array}{l}\text { Adjusted OR* } \\
\mathbf{( 9 5 \% ~ C l )}\end{array}$ \\
\hline Primary exposure & & \\
\hline $\begin{array}{l}\text { AD use from 1DG to index } \\
\text { date† }\end{array}$ & $1.36(1.28$ to 1.45$)$ & $1.19(1.08$ to 1.30) \\
\hline
\end{tabular}

\section{Covariates}

As of first day of gestation

\begin{tabular}{|lll|}
\hline $\begin{array}{l}\text { Age (years) } \\
<18\end{array}$ & $0.49(0.41$ to 0.59$)$ & 0.46 (0.38 to 0.55$)$ \\
\hline $18-34$ & 1.00 (Reference) & 1.00 (Reference) \\
\hline$>34$ & $2.01(1.95$ to 2.08$)$ & $1.98(1.92$ to 2.05$)$ \\
\hline Urban residence & $1.16(1.11$ to 1.20$)$ & $1.09(1.05$ to 1.14$)$ \\
\hline Recipient of social assistance & $1.24(1.20$ to 1.28$)$ & $1.23(1.19$ to 1.28$)$ \\
\hline $\begin{array}{l}\text { AD use during } 6 \text { months before } \\
\text { gestation }\end{array}$ & $1.29(1.22$ to 1.36$)$ & $1.07(0.98$ to 1.17$)$ \\
\hline
\end{tabular}

6 months before gestation

\begin{tabular}{|c|c|c|}
\hline \multicolumn{3}{|l|}{ Maternal comorbidities } \\
\hline Depression/anxiety & 1.10 (1.04 to 1.16$)$ & $0.93(0.88$ to 1.00$)$ \\
\hline $\begin{array}{l}\text { Chronic/gestational } \\
\text { hypertension }\end{array}$ & 1.83 (1.66 to 2.02$)$ & 1.51 (1.37 to 1.67$)$ \\
\hline Asthma & 1.18 (1.12 to 1.25$)$ & $1.13(1.07$ to 1.19$)$ \\
\hline Cardiovascular diseases $\ddagger$ & 1.16 (1.05 to 1.29$)$ & 1.05 (0.94 to 1.16$)$ \\
\hline Thyroid disorders & 1.48 (1.38 to 1.59$)$ & 1.35 (1.25 to 1.45$)$ \\
\hline Polycystic ovarian syndrome & 2.34 (1.41 to 3.88$)$ & 2.09 (1.25 to 3.49$)$ \\
\hline \multicolumn{3}{|l|}{ Medication use } \\
\hline \multicolumn{3}{|c|}{$\begin{array}{l}\text { Other medications excluding ADs and medications related to maternal } \\
\text { comorbidities }\end{array}$} \\
\hline $1-2$ & 1.05 (1.02 to 1.10$)$ & 1.03 (0.99 to 1.07$)$ \\
\hline$>2$ & 1.06 (1.02 to 1.09$)$ & $1.02(0.98$ to 1.05$)$ \\
\hline \multicolumn{3}{|l|}{ Healthcare services utilisation } \\
\hline Visit to psychiatrists & $1.19(1.09$ to 1.29$)$ & 1.03 (0.94 to 1.13$)$ \\
\hline Visit to physicians & 1.10 (1.07 to 1.13 ) & 1.05 (1.02 to 1.09$)$ \\
\hline $\begin{array}{l}\text { Hospitalisations or } \\
\text { emergency department visits }\end{array}$ & $1.01(1.00$ to 1.05$)$ & 0.97 (0.93 to 1.01$)$ \\
\hline
\end{tabular}

*Adjusted for variables (a) on first day of gestation (maternal age, area of residence (urban vs rural), receipt of social assistance during pregnancy); (b) during 6 months before gestation (physician-based diagnoses or filled prescriptions of related medications for chronic comorbidities (hypertension, asthma and thyroid disorders); physician-based diagnoses of maternal diseases (depression, cardiovascular diseases and polycystic ovarian syndrome); medication use other than antidepressants and drugs for hypertension, asthma and thyroid disorders and visits to psychiatrists); history of antidepressant use and health service utilisation (visits to physicians and hospitalisations or emergency department visits).

†Exposure to any antidepressant versus no antidepressant use as reference. $\ddagger$ Cardiovascular diseases (ischaemic heart disease; pulmonary heart disease and diseases of pulmonary circulation; other forms of heart disease; cerebrovascular diseases; diseases of arteries, arterioles and capillaries; diseases of veins, lymphatic vessels and lymph nodes, not elsewhere classified; other and unspecified disorders of the circulatory system; diseases of the circulatory system complicating pregnancy, childbirth and the puerperium; abnormal results of cardiovascular function studies).

$A D$, antidepressant; 1DG, first day of gestation.

pathway. In addition, still in animal studies, serotonin has been shown to play a role in the compensation mechanism, which is established following natural insulin resistance in pregnancy. ${ }^{47} \mathrm{AD}$ use inhibits the insulin pathway resulting in insulin resistance and potentially GDM. The effects of ADs on glucose dysregulation and weight gain seem stronger with TCAs (especially amitriptyline) than with SNRIs and SSRIs due to the varying affinity for serotoninergic, noradrenergic and histamine receptors. ${ }^{14} 48$ A study reported that, due to their high affinity for the serotonin reuptake transporter, SSRIs could generate hypoglycaemia whereas ADs with high affinity for the 5-HT2c receptor (serotonin), H1-receptor (histamine) and norepinephrine reuptake such as amitriptyline and venlafaxine could lead to hyperglycaemia. ${ }^{17}$ Moreover, the study added that, among their cases, glucose disturbances were occurring within 1 month of treatment and that cumulative effects of $\mathrm{AD}$ drugs on glucose control were possible, especially with a longer duration of treatment. ${ }^{17}$

It has also been proposed that, in the short term, SSRIs may have a neutral weight effect or even reduce weight gain (fluoxetine) and therefore improve diabetes, which could partly explain our results. ${ }^{49}$ Our study confirms that $\mathrm{ADs}$ have heterogeneous effects regarding GDM incidence, which we believe is mainly due to their varying pharmacological properties.

\section{Strengths of the study}

To our knowledge, this is the first study looking specifically at $\mathrm{AD}$ classes and duration of use during pregnancy and the risk of GDM. With 17 years of follow-up, we were able to investigate both class and individual drug effects of ADs. Furthermore, in the QPC, data are prospectively collected and provide valid and accurate information on filled prescriptions and physician-based diagnoses which rule out the potential for recall bias and ascertainment bias. The use of a nested case-control design allows account to be taken of the time-varying nature of our exposure. Finally, our definition of GDM based on diagnoses and prescriptions filled for antidiabetic drugs has been validated and used in previous studies.

\section{Limitations of the study}

Residual confounding by indication related to the underlying depression cannot be ruled out. Our study was not adjusted for depression during pregnancy because depression during pregnancy could be on the causal pathway between AD use and GDM. When we restricted our cohort to a subgroup of pregnancies diagnosed with depression/anxiety before pregnancy, our sensitivity analysis supports our main results. This also minimises the possible detection bias because all pre-pregnancy depressed women might have similar contact with healthcare professionals and an equal chance to be diagnosed for additional comorbidities. Also, since the screening for GDM is part of the standard pregnancy monitoring in Quebec, a detection bias is less likely to occur. To further control for confounding by disease severity, we adjusted for documented proxies for depression severity such as visits to the psychiatrist and co-medication, including medications for mental diseases such as benzodiazepines and antipsychotics. 
Table 3 Cumulative duration of exposure to antidepressants and risk of gestational diabetes

\begin{tabular}{|c|c|c|c|c|}
\hline \multirow[b]{2}{*}{ Exposure status } & \multirow[b]{2}{*}{ Cases $(n=20905)$} & \multirow[b]{2}{*}{ Controls $(n=209048)$} & \multicolumn{2}{|l|}{ OR (95\% Cl) } \\
\hline & & & Crude & Adjusted $^{*}$ \\
\hline No exposure & $19753(94.5)$ & 200461 (95.9) & 1.00 (Reference) & 1.00 (Reference) \\
\hline \multicolumn{5}{|c|}{ Cumulative duration (d) in days among SSRI users } \\
\hline$<90$ & $242(1.2)$ & $2047(0.9)$ & $1.20(1.05$ to 1.37$)$ & 1.09 (0.94 to 1.27$)$ \\
\hline $90 \leq d<180$ & $83(0.4)$ & $765(0.4)$ & 1.10 (0.88 to 1.38$)$ & 0.98 (0.78 to 1.25$)$ \\
\hline $180 \leq d$ & $210(1.0)$ & $1674(0.8)$ & $1.27(1.10$ to 1.47$)$ & $1.11(0.94$ to 1.32$)$ \\
\hline \multicolumn{5}{|c|}{ Cumulative duration (d) in days among SNRI users } \\
\hline$<90$ & $78(0.4)$ & $718(0.3)$ & $1.10(0.88$ to 1.39$)$ & $1.02(0.80$ to 1.30$)$ \\
\hline $90 \leq \mathrm{d}<180$ & $29(0.1)$ & $239(0.1)$ & $1.23(0.84$ to 1.80$)$ & $1.15(0.78$ to 1.70$)$ \\
\hline $180 \leq d$ & $123(0.6)$ & $707(0.3)$ & 1.76 (1.45 to 2.13 ) & 1.58 (1.28 to 1.95$)$ \\
\hline \multicolumn{5}{|c|}{ Cumulative duration (d) in days among TCA users } \\
\hline$<90$ & $108(0.5)$ & $666(0.3)$ & 1.64 (1.34 to 2.00$)$ & $1.42(1.15$ to 1.75$)$ \\
\hline $90 \leq \mathrm{d}<180$ & $21(0.1)$ & $89(0.04)$ & 2.39 (1.49 to 3.83$)$ & $1.96(1.21$ to 3.17$)$ \\
\hline $180 \leq d$ & $14(0.07)$ & $73(0.03)$ & 1.94 (1.10 to 3.43$)$ & $1.46(0.82$ to 2.61$)$ \\
\hline \multicolumn{5}{|c|}{ Cumulative duration (d) in days among users of other antidepressants } \\
\hline$<90$ & $49(0.2)$ & $440(0.2)$ & $1.13(0.84$ to 1.52$)$ & $0.99(0.73$ to 1.34$)$ \\
\hline $90 \leq \mathrm{d}<180$ & $12(0.06)$ & $56(0.03)$ & 2.16 (1.16 to 4.00$)$ & $1.52(0.81$ to 2.86$)$ \\
\hline $180 \leq d$ & $14(0.07)$ & $103(0.05)$ & $1.38(0.79$ to 2.41$)$ & $1.10(0.62$ to 1.94$)$ \\
\hline \multicolumn{5}{|c|}{ Cumulative duration (d) in days among combined users (users of $\geq 2$ antidepressants) } \\
\hline$<90$ & $64(0.3)$ & $399(0.2)$ & $1.62(1.24$ to 2.10$)$ & $1.31(0.99$ to 1.73$)$ \\
\hline $90 \leq \mathrm{d}<180$ & $42(0.2)$ & $280(0.1)$ & $1.52(1.10$ to 2.10$)$ & $1.32(0.94$ to 1.84$)$ \\
\hline $180 \leq d$ & $63(0.3)$ & $331(0.2)$ & 1.92 (1.47 to 2.50$)$ & 1.56 (1.18 to 2.07$)$ \\
\hline
\end{tabular}

*Adjusted for variables (a) on first day of gestation (maternal age, area of residence (urban vs rural), receipt of social assistance during pregnancy); (b) during 6 months before gestation (physician-based diagnoses or filled prescriptions of related medications for chronic comorbidities (hypertension, asthma and thyroid disorders); physician-based diagnoses of maternal diseases (depression, cardiovascular diseases and polycystic ovarian syndrome); medication use other than antidepressants and drugs for hypertension, asthma and thyroid disorders and visits to psychiatrists); history of antidepressant use and health service utilisation (visits to physicians and hospitalisations or emergency department visits).

We included late cases of GDM recorded after 28 weeks of gestation, which could lead to a misclassification of the exposure and result in an underestimation of the effect. However, when we considered only cases of GDM with a diagnosis between 24 and 28 weeks of gestation, as recommended by the Canadian guidelines, our results were similar to our main analyses. Although we adjusted for most risk factors for GDM, some lifestyle variables such as smoking, weight gain, BMI and physical activity practise are missing in our database, which could result in residual confounding. Previous studies have found inconsistent results for the association between smoking and GDM, but some have shown an association. ${ }^{50}{ }^{51}$ In a sensitivity analysis, we restricted our cohort to women with depression before pregnancy and found consistent results with the main analysis. This restriction de facto gives us a population of pregnant women with similar characteristics regarding lifestyles such as smoking, alcohol intake and physical activity. We are confident that these missing variables in our analyses would minimally affect our results because Reis and Källén in their study adjusted their analyses for BMI and smoking and found consistent estimates with ours. ${ }^{23}$ Family income and education were not directly taken into account in our study. However, women covered by the public prescription drug plan are women of low to middle socioeconomic status. In our analysis, we adjusted for the adherent status with the public prescription drug plan, which is a proxy for the socioeconomic status and education. Adherent women have higher socioeconomic status than beneficiaries. Therefore, by adjusting for adherent status, we also partially adjusted for education. All these sensitivity analyses show that our estimates are stable and that, if residual confounding is present, it is minimal. Moreover, we could not adjust for ethnicity in our study because this variable is not available in our databases. However, a previous study performed on the QPC based on self-administered questionnaires collected information not present in our administrative databases such as ethnicity. ${ }^{52}$ This study found that $88.5 \%$ of the women in the QPC were from a Caucasian background. Current evidence suggests that women from a Caucasian ethnic background had the lowest risk of GDM 


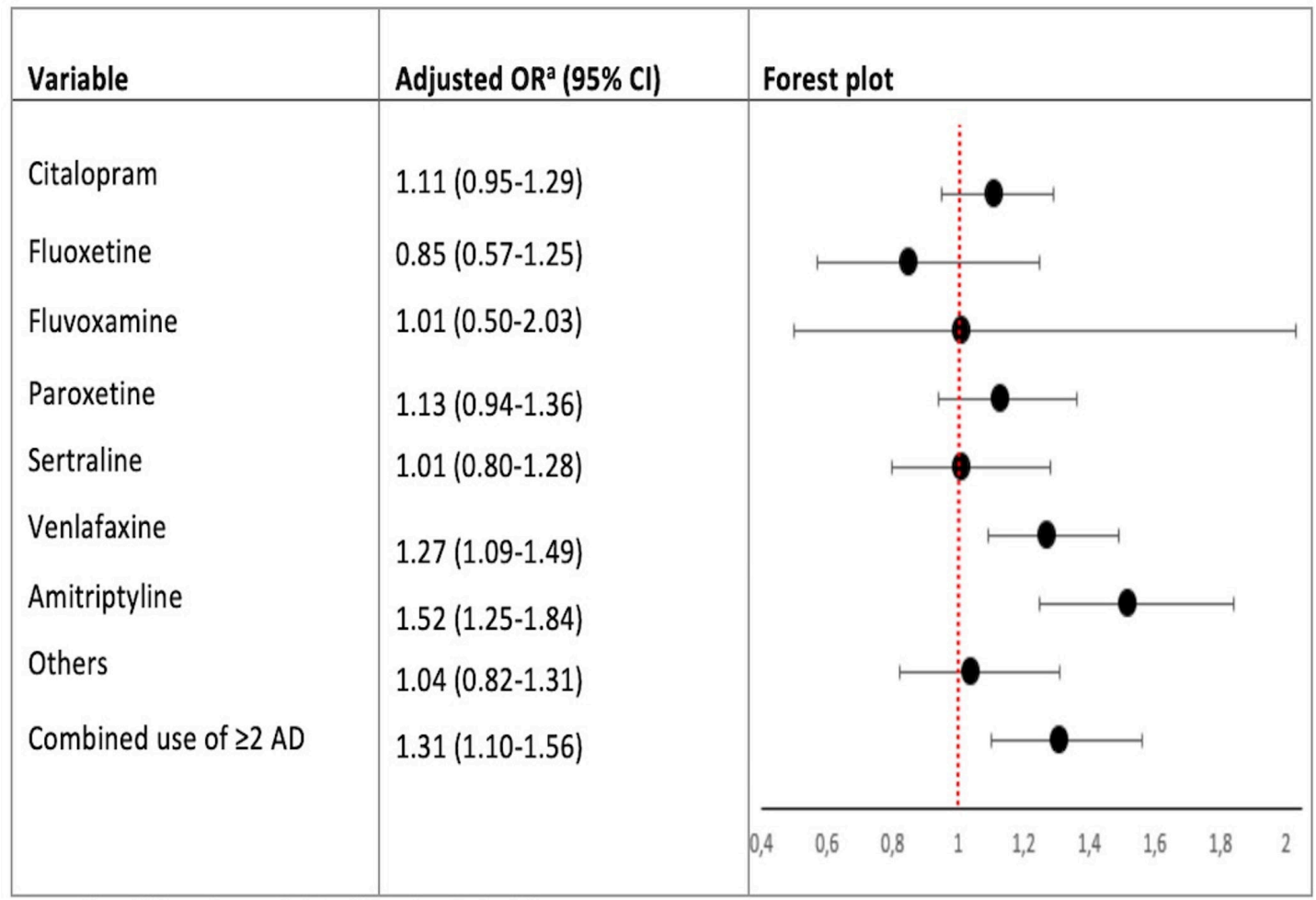

Note: $\mathrm{Cl}$ confidence interval; OR odds ratio; AD Antidepressant;

${ }^{a}$ Adjusted for variables (a) on first day of gestation (maternal age, area of residence [urban v. rural], receipt of social assistance during pregnancy); (b) During 6 months before gestation (physician -based diagnoses or filled prescriptions of related medications for chronic comorbidities [hypertension, asthma and thyroid disorders]; physician-based diagnoses of maternal diseases [depression, cardiovascular diseases and polycystic ovarian syndrome]; medication use others than antidepressants and drugs use for hypertension, asthma and thyroids disorders and visits to psychiatrists); history of antidepressants use and health services utilization [visits to physicians and hospitalisations or emergency department visit].

Figure 2 Types of antidepressant use and risk of gestational diabetes.

compared with other ethnic groups. ${ }^{53}$ Thus, our estimates are calculated in a majority Caucasian population and are likely underestimations. This does not affect the internal validity of our study, but might partly limit its generalisability to other ethnic groups. Finally, generalisability to overweight/obese women or women with cystic fibrosis could be affected, given that our study includes only pregnancies of women without cystic fibrosis and non-overweight/non-obese women. Nevertheless, this does not affect the internal validity of our study. Moreover, in a previous study, socioeconomic status was shown to be an effect modifier in the QPC, and those women on the public drug coverage plan and women on private drug insurance have similar characteristics and comorbidities. ${ }^{54}$

Overall, AD use specifically in pregnant women of normal weight was associated with an increased risk of GDM as well as with a cumulative duration effect, after taking into account underlying maternal depression. Amitriptyline and venlafaxine were associated with an increased risk of GDM.

The treatment of depression during pregnancy is a major concern and is challenging because depression is prevalent before and during pregnancy, and untreated depression can lead to relapse during pregnancy and in the postpartum period ${ }^{55}$ Hence, adverse outcomes associated with $\mathrm{AD}$ use during pregnancy including GDM should be weighed against the consequences of non-medicated depression, especially for women with severe depression. Further studies are needed to replicate our findings. Results from more studies could translate into changes in clinical practice guidelines. Nevertheless, our findings raise awareness of the risk of GDM with the use of specific ADs during pregnancy.

Contributors All authors participated in the study conception and design. AB acquired the data. MD and OS performed the analyses. MD wrote the manuscript and all authors participated in the interpretation of the results and critical revision of the manuscript. $A B$ is the guarantor of this work and, as such, had full access to all the data in the study and takes responsibility for the integrity of the data and the accuracy of the data analysis.

Funding This study was funded by the Canadian Institutes of Health Research (CIHR) - CAN-AIM grant; Fonds de la recherche du Québec-Santé (FRQ-S) - Réseau de recherche sur les médicaments RQRM-AbbVie. The funding body had no role in the design and conduct of the study; collection, management, analysis and interpretation of the data; preparation, review or approval of the manuscript; or the decision to submit the manuscript for publication.

Competing interests $A B$ has served as a consultant for plaintiffs in litigations involving antidepressants and birth defects. MD and OS report no conflicts of interest; no other financial relationships with any organisations that might have an interest in the submitted work in the previous three years; and no other 
relationships or activities that could appear to have influenced the submitted work.

Patient consent for publication Not required.

Ethics approval The study was approved by the Sainte-Justine's Hospital Ethics Committee (\#1740). The Quebec "Commission d'accès à l'information" authorized database linkages (\#2976).

Provenance and peer review Not commissioned; externally peer reviewed.

Data availability statement No data are available. All data relevant to the study are included in the article or uploaded as supplementary information.

Open access This is an open access article distributed in accordance with the Creative Commons Attribution Non Commercial (CC BY-NC 4.0) license, which permits others to distribute, remix, adapt, build upon this work non-commercially, and license their derivative works on different terms, provided the original work is properly cited, appropriate credit is given, any changes made indicated, and the use is non-commercial. See: http://creativecommons.org/licenses/by-nc/4.0/.

\section{REFERENCES}

1. Thompson D, Berger H, Feig D, et al. Diabetes and pregnancy. Can $J$ Diabetes 2013;37(Suppl 1):S168-S183.

2. Hod M, Kapur A, Sacks DA, et al. The International Federation of Gynecology and Obstetrics (FIGO) initiative on gestational diabetes mellitus: a pragmatic guide for diagnosis, management, and care. Int $J$ Gynecol Obstet 2015;131:S173-S211.

3. Chun L, Healy-Profitos J, Tu MT, et al. Évolution du diabète gestationnel au Québec de 1989 à 2012: rapport de surveillance. INSPQ, 2017.

4. Feig DS, Hwee J, Shah BR, et al. Trends in incidence of diabetes in pregnancy and serious perinatal outcomes: a large, populationbased study in Ontario, Canada, 1996-2010. Diabetes Care 2014;37:1590-6.

5. Zhu Y, Zhang C. Prevalence of gestational diabetes and risk of progression to type 2 diabetes: a global perspective. Curr Diab Rep 2016;16:7.

6. Jenum AK, Mørkrid K, Sletner L, et al. Impact of ethnicity on gestational diabetes identified with the WHO and the modified International Association of Diabetes and Pregnancy Study Groups criteria: a population-based cohort study. Eur J Endocrinol 2012;166:317-24.

7. Brunner S, Stecher L, Ziebarth S, et al. Excessive gestational weight gain prior to glucose screening and the risk of gestational diabetes: a meta-analysis. Diabetologia 2015;58:2229-37.

8. Metzger BE, Lowe LP, Dyer AR, et al. Hyperglycemia and adverse pregnancy outcomes. N Engl J Med 2008;358:1991-2002.

9. Clausen TD, Mathiesen ER, Hansen T, et al. High prevalence of type 2 diabetes and pre-diabetes in adult offspring of women with gestational diabetes mellitus or type 1 diabetes: the role of intrauterine hyperglycemia. Diabetes Care 2008;31:340-6.

10. Fraser A, Lawlor DA. Long-term health outcomes in offspring born to women with diabetes in pregnancy. Curr Diab Rep 2014;14:489.

11. Tobias DK, Stuart JJ, Li S, et al. Association of history of gestational diabetes with long-term cardiovascular disease risk in a large prospective cohort of US women. JAMA Intern Med 2017;177:1735-42.

12. Huybrechts KF, Palmsten $\mathrm{K}$, Mogun $\mathrm{H}$, et al. National trends in antidepressant medication treatment among publicly insured pregnant women. Gen Hosp Psychiatry 2013;35:265-71.

13. Serretti A, Mandelli L. Antidepressants and body weight: a comprehensive review and meta-analysis. J Clin Psychiatry 2010;71:1259-72.

14. Derijks HJ, Meyboom RHB, Heerdink ER, et al. The association between antidepressant use and disturbances in glucose homeostasis: evidence from spontaneous reports. Eur J Clin Pharmacol 2008;64:531-8.

15. Deuschle M. Effects of antidepressants on glucose metabolism and diabetes mellitus type 2 in adults. Curr Opin Psychiatry 2013;26:60-5.

16. Hennings JM, Schaaf L, Fulda S. Glucose metabolism and antidepressant medication. Curr Pharm Des 2012:18:5900-19.

17. Khoza S, Barner JC. Glucose dysregulation associated with antidepressant agents: an analysis of 17 published case reports. Int $J$ Clin Pharm 2011;33:484-92.

18. Kessler RC, Berglund P, Demler O, et al. The epidemiology of major depressive disorder: results from the National Comorbidity Survey Replication (NCS-R). JAMA 2003;289:3095-105.
19. Luppino FS, de Wit LM, Bouvy PF, et al. Overweight, obesity, and depression: a systematic review and meta-analysis of longitudinal studies. Arch Gen Psychiatry 2010;67:220-9.

20. John U, Meyer C, Rumpf H-J, et al. Relationships of psychiatric disorders with overweight and obesity in an adult general population. Obes Res 2005;13:101-9.

21. Molyneaux E, Poston L, Khondoker M, et al. Obesity, antenatal depression, diet and gestational weight gain in a population cohort study. Arch Womens Ment Health 2016;19:899-907.

22. Nørgaard M, Ehrenstein V, Vandenbroucke JP. Confounding in observational studies based on large health care databases: problems and potential solutions - a primer for the clinician. Clin Epidemiol 2017;9:185-93.

23. Reis M, Källén B. Delivery outcome after maternal use of antidepressant drugs in pregnancy: an update using Swedish data. Psychol Med 2010;40:1723-33.

24. Wen SW, Yang Q, Garner P, et al. Selective serotonin reuptake inhibitors and adverse pregnancy outcomes. Am J Obstet Gynecol 2006;194:961-6.

25. Bérard A, Sheehy O. The Quebec Pregnancy Cohort--prevalence of medication use during gestation and pregnancy outcomes. PLOS One 2014;9:e93870.

26. Vilain A, Otis S, Forget $\mathrm{A}$, et al. Agreement between administrative databases and medical charts for pregnancy-related variables among asthmatic women. Pharmacoepidemiol Drug Saf 2008;17:345-53.

27. Zhao J-P, Sheehy O, Gorgui J, et al. Can we rely on pharmacy claims databases to ascertain maternal use of medications during pregnancy? Birth Defects Res 2017;109:423-31.

28. Xiang $\mathrm{AH}$, Takayanagi $\mathrm{M}$, Black $\mathrm{MH}$, et al. Longitudinal changes in insulin sensitivity and beta cell function between women with and without a history of gestational diabetes mellitus. Diabetologia 2013;56:2753-60.

29. Andrade SE, Reichman ME, Mott K, et al. Use of selective serotonin reuptake inhibitors (SSRIs) in women delivering liveborn infants and other women of child-bearing age within the U.S. Food and Drug Administration's Mini-Sentinel program. Arch Womens Ment Health 2016:19:969-77.

30. Baribeau V, Beauchesne M-F, Rey Évelyne, et al. The use of asthma controller medications during pregnancy and the risk of gestational diabetes. J Allergy Clin Immunol 2016;138:1732-3.

31. Jelin AC, Sharshiner R, Caughey AB. Maternal co-morbidities and neonatal outcomes associated with cystic fibrosis. $J$ Matern Fetal Neonatal Med 2017;30:4-7.

32. Ernster VL. Nested case-control studies. Prev Med 1994;23:587-90.

33. Essebag V, Genest J Jr, Suissa S, et al. The nested case-control study in cardiology. Am Heart J 2003;146:581-90.

34. Essebag V, Platt RW, Abrahamowicz M, et al. Comparison of nested case-control and survival analysis methodologies for analysis of time-dependent exposure. BMC Med Res Methodol 2005;5:5.

35. Blais L, Kettani F-Z, Forget A. Associations of maternal asthma severity and control with pregnancy complications. J Asthma 2014:51:391-8.

36. Bowker SL, Savu A, Lam NK, et al. Validation of administrative data case definitions for gestational diabetes mellitus. Diabet Med 2017;34:51-5.

37. Egan AM, Vellinga A, Harreiter $\mathrm{J}$, et al. Epidemiology of gestational diabetes mellitus according to IADPSG/WHO 2013 criteria among obese pregnant women in Europe. Diabetologia 2017;60:1913-21.

38. Liang K-YEE, Zeger SL. Longitudinal data analysis using generalized linear models. Biometrika 1986;73:13-22.

39. Barnard K, Peveler RC, Holt RIG. Antidepressant medication as a risk factor for type 2 diabetes and impaired glucose regulation: systematic review. Diabetes Care 2013;36:3337-45.

40. Salvi V, Grua I, Cerveri G, et al. The risk of new-onset diabetes in antidepressant users - a systematic review and meta-analysis. PLOS One 2017;12:e0182088.

41. Yoon JM, Cho E-G, Lee H-K, et al. Antidepressant use and diabetes mellitus risk: a meta-analysis. Korean J Fam Med 2013;34:228-40.

42. Andersohn F, Schade R, Suissa S, et al. Long-term use of antidepressants for depressive disorders and the risk of diabetes mellitus. Am J Psychiatry 2009;166:591-8.

43. Burcu M, Zito JM, Safer DJ, et al. Association of antidepressant medications with incident type 2 diabetes among Medicaid-insured youths. JAMA Pediatr 2017;171:1200-7.

44. Isaac R, Boura-Halfon S, Gurevitch D, et al. Selective serotonin reuptake inhibitors (SSRIs) inhibit insulin secretion and action in pancreatic $\beta$ cells. J Biol Chem 2018;293:4577-8.

45. Levkovitz Y, Ben-Shushan G, Hershkovitz A, et al. Antidepressants induce cellular insulin resistance by activation of IRS-1 kinases. Mol Cell Neurosci 2007;36:305-12. 
46. Carvalho F, Barros D, Silva J, et al. Hyperglycemia induced by acute central fluoxetine administration: role of the central $\mathrm{CRH}$ system and 5-HT3 receptors. Neuropeptides 2004;38:98-105.

47. Baeyens L, Hindi S, Sorenson RL, et al. $\beta$-Cell adaptation in pregnancy. Diabetes Obes Metab 2016;18(Suppl 1):63-70.

48. Salvi V, Mencacci C, Barone-Adesi F. H1-Histamine receptor affinity predicts weight gain with antidepressants. Eur Neuropsychopharmacol 2016;26:1673-7.

49. Harvey BH, Bouwer CD. Neuropharmacology of paradoxic weight gain with selective serotonin reuptake inhibitors. Clin Neuropharmacol 2000;23:90-7.

50. Hosler AS, Nayak SG, Radigan AM. Stressful events, smoking exposure and other maternal risk factors associated with gestational diabetes mellitus. Paediatr Perinat Epidemiol 2011;25:566-74.
51. Terry PD, Weiderpass E, Ostenson C-G, et al. Cigarette smoking and the risk of gestational and pregestational diabetes in two consecutive pregnancies. Diabetes Care 2003;26:2994-8.

52. Bérard A, Sheehy O. The Quebec Pregnancy Cohort--prevalence of medication use during gestation and pregnancy outcomes. PLOS One 2014;9:e93870-e70.

53. Hedderson MM, Darbinian JA, Ferrara A. Disparities in the risk of gestational diabetes by race-ethnicity and country of birth. Paediatr Perinat Epidemiol 2010;24:441-8.

54. Bérard A, Lacasse A. Validity of perinatal pharmacoepidemiologic studies using data from the RAMQ administrative database. Can $J$ Clin Pharmacol 2009;16:e360-9.

55. Howard L, Hoffbrand SE, Henshaw C, et al. Antidepressant prevention of postnatal depression. Cochrane Database Syst Rev 2005;66. 